\title{
Test Object for Accurate and Reproducible Measurement of the Detection Response of Hand-worn and Hand-held Metal Detectors
}

\author{
Nicholas G. Paulter, Jr. ${ }^{1}$, Donald R. Larson ${ }^{2}$, and John A. Ely ${ }^{3}$ \\ ${ }^{1}$ National Institute of Standards and Technology, \\ Gaithersburg, MD 20899 \\ ${ }^{2}$ Entegra Corporation, \\ Aurora, CO 80016 \\ ${ }^{3}$ Correctional Security Consultant, \\ Mt. Airy, MD 21771 \\ nicholas.paulter@nist.gov \\ donald.larson@entegra-corp.com \\ jely60@comcast.net
}

\begin{abstract}
The assessment of the detection performance of metal detectors (hand-worn, hand-held, and walk-through models) is based on the ability of the detectors to sound an alarm when presented with a test object. These test objects are typically actual threat items or simulated threat items. The orientation of these test objects with the magnetic field generated by the metal detectors may affect the detectability of the test objects. More importantly, small misorientations of a threat object or simulated threat object may cause the operator to incorrectly attribute to the metal detector a higher performance than it is capable of providing. Consequently, to support accurate and reproducible characterization of the performance of a metal detector, orientation effects should be minimized or eliminated. We discuss the use of spherical test objects to eliminate this potentially serious error in the assessment of the detection performance of a metal detector. In this study, we consider only hand-worn and hand-held metal detectors because of their similarity in size and operation.
\end{abstract}

Key words: detection response; hand-held metal detector; hand-worn metal detector; magnetic field; object orientation; test objects.

Accepted: August 26, 2016

Published: September 6, 2016

http://dx.doi.org/10.6028/jres.121.019

\section{Introduction}

Security metal detectors work by generating an alternating magnetic field that interacts with electrically conducting and/or magnetically permeable objects that are nearby, and then sensing the effect of that interaction. Hand-held metal detectors (HHMDs) are ubiquitous in security screening of metal threat objects and contraband. Hand-worn metal detectors (HWMDs) are relatively new instantiation of security screening metal detector. These HWMDs may be full-glove or half-glove models or simply a strap containing a sensor that lies against the palm side of the hand. HWMDs and HHMDs are intended to be used in close proximity to the subject being searched: the HWMD typically operates with the sensor within a couple of millimeters from the surface being searched, whereas the HHMD is nominally held $1 \mathrm{~cm}$ to $2 \mathrm{~cm}$ from the surface being scanned, depending on the intent of the search.

To test the detection performance of HHMDs and HWMDs, metal test objects are brought near the metal detector and the presence or absence of an alarm is recorded. These metal test objects are often an 
actual threat object. However, the design or manufacture of an actual threat object may change unbeknownst to the operator, and thus change the apparent sensitivity of a metal detector to that threat object. The purposes of the test objects include to assess the performance of a given model of metal detectors, to perform a historical comparison of the performance of a given metal detector or model of metal detectors, and to perform a product comparison. To do these functions accurately and reproducibly, the test object should be well defined and stable. This is where an exemplar that emulates the electromagnetic response of an actual threat should be used. These exemplars do not need to look like an actual threat object, but they should have well defined geometries and material characteristics, with appropriate tolerances, such that the exemplar and threat have nominally the same detection signature. Such exemplars allow more consistent comparative evaluation of the performance of metal detectors than will actual threat objects. Such exemplars should be the basis of a minimally acceptable performance requirement for a metal detector. Before being considered for deployment, a metal detector should meet the detection requirements for a set of exemplars, each testing for a different threat.

This article discusses the effect of a test object (an exemplar) on the measured performance of a metal detector. To have an appreciation of how the exemplar affects this performance measure, a brief introduction to the uncertainty in measurement is required. Measurements are subject to various factors that introduce some amount of uncertainty into their result. These measurement uncertainties give information on the confidence a person may have on the measurement results. For metal detectors, the measurement uncertainty, $u_{\text {meas }}$, can be described by the following formula using the method of linear propagation of uncertainties [1]:

$$
u_{\text {meas }}=\sqrt{u_{\text {inst }}^{2}+u_{R \& R}^{2}+u_{\text {exmplr }}^{2}},
$$

where $u_{\text {inst }}$ is the measurement uncertainty for all the instruments, analyses, and computational methods used; $u_{R \& R}$ is the uncertainty for reproducibility and repeatability, which includes contributions such as operator and environmental effects; and $u_{\text {exmplr }}$ are the measurement uncertainties associated with the exemplar, which has other contributors:

$$
u_{\text {exmplr }}=\sqrt{u_{\text {prpty }}^{2}+u_{\text {geom }}^{2}+u_{\text {orient }}^{2}} \text {, }
$$

where $u_{\text {prpty }}$ is the measurement uncertainty associated with material properties of the test object, where the two material properties that are of concern are the electrical conductivity, $\sigma$, and the magnetic permeability, $\mu$; $u_{\text {geom }}$ is the uncertainty for the physical dimensions of the test object; and $u_{\text {orient }}$ is the uncertainty associated with the orientation of the test object relative to the metal detector under test.

The values of $u_{\text {inst }}$ and $u_{R \& R}$ are typically less than a couple percent of the measured value, and so will not adversely affect the ability to assess the detection performance of a metal detector. Moreover, these uncertainties will be the same regardless of the exemplar being used and will not be discussed further in this study. The uncertainties, $u_{\text {prpty }}$ and $u_{\text {geom }}$ are dependent on the exemplar, but will not vary with its design: uncertainties in geometries are fixed by machining tolerances and uncertainties in material properties are fixed by the selection of the materials and are independent of design. The only measurement uncertainty contribution that can be noticeably controlled during a measurement is $u_{\text {orient }}$, which will be discussed in detail later.

\section{Metal Detection Exemplars}

Metal detector exemplars, to date, have been fabricated from a single material machined to resemble the threat object. The dimensions of the exemplar are adjusted so that its detection signature experimentally conforms to the signature of the threat item. For example, for handgun threats, the exemplars are L-shaped metal objects; for knives, the exemplar is a metal rectangular prism, etc. The exemplars currently in use are 
derived from the National Institute of Justice (NIJ) HHMD standard [2] as developed for the NIJ by the National Institute of Standards and Technology. These exemplars are shown in Appendix A.

The materials, materials properties, dimensions, and tolerances on the properties and dimensions of the exemplars are given in Appendix A. The materials are specified using the Unified Numbering System (UNS) convention (see Appendix B). The UNS designation primarily addresses composition and not the electromagnetic material properties. Consequently, the electromagnetic properties, specifically electrical conductivity and magnetic permeability, are also specified. Because the geometries, material of construction, and electromagnetic properties for the exemplars are fixed and within specified tolerances, these exemplars provide superior performance compared to actual threat items for accurate and reproducible characterization and assessment of the detection capability of metal detectors. The current shapes of the exemplars resemble the actual threat items, and this was done to assure operators that their actual threat objects would be detected. However, these resemblances weaken the reproducibility and accuracy of detection performance testing, performance tracking, and product comparison. Moreover, this restriction is unnecessary for the purpose of a metal detector minimum performance standard.

Metal detectors are tested to ensure that they meet the requirements of different performance classifications, typically based on either threat level or object size. Threat-level classifications may be ambiguous; for example, a razor blade is not considered a high security threat for events involving very important persons (because of access to the person) but it is considered a high security threat in correctional environments. The ASTM standard, on the other hand, uses object size for classification. Although this classification scheme is not perfect because of the effect of material properties, it is a more logical classification scheme than threat level.

Figures A1 through A7 in Appendix A show a set of exemplars currently used in the NIJ standards and that has been proposed for the ASTM HWMD standard. The physical dimensions of these exemplars are adjusted based on the material properties and the actual threat they are intended to emulate. For example, consider the handgun exemplar (Fig. A1). The steel (UNS G41400) handgun exemplar is the smallest of the three handgun exemplars because the magnetic field generated by a metal detector couples more effectively to the steel exemplar than to the other two (aluminum and zinc). Consequently, the steel exemplar does not have to be as large as the other two to generate a nominally equivalent detection signal. In Fig. A2, a medium-sized test object, the knife exemplar fabricated from either aluminum (UNS A95052) or steel (UNS G41300), is shown. If the material of construction for the knife exemplar is a nonferromagnetic stainless steel (UNS S30400), for which the relative permeability is approximately one and the electrical conductivity is small, the knife exemplar would be considered a small-sized test object. Therefore, two knife exemplars with the same dimensions can be classified as either a medium-sized or small-sized test object depending on their electromagnetic properties. More importantly, a small misorientation of the small-sized test object from a reference orientation can cause a detection signal similar to a medium-sized test object. This is the main focus of the remainder of this article.

\section{Test Object Orientation}

Although the use of the current exemplars for metal detector testing has many advantages over actual threats, one critical aspect of the exemplars that has not been addressed is its orientational uncertainty relative to the magnetic field generated by the metal detector during testing. This orientational uncertainty is a consequence of the geometric anisotropy of the current exemplar designs, and can lead to erroneous claims of superior detection performance. The orientational uncertainty has several sources, which can be described using the method of linear propagation of uncertainties (LPU) by:

$$
u_{\text {orient }}=\sqrt{u_{x m p / b l k}^{2}+u_{b l k / h l d r 1}^{2}+u_{h l d r 1 / \text { rob }}^{2}+u_{\text {rob/hldr } 2}^{2}+u_{\text {hldr } 2 / d c t r}^{2}+\sigma_{\theta}^{2}} \text {, }
$$

where $u_{x m p / l k}$ is the uncertainty in the orientation of a reference plane or position on the exemplar relative to a reference plane on the block, where the block is used to contain the exemplar; $u_{b l k h l d r 1}$ is the uncertainty in the orientation of the block relative to the block holder, where the block holder is a device that secures the 
block to the robotic or manual positioning instrument; $u_{h l d r 1 / \text { rob }}$ is the uncertainty in the orientation of the block holder relative to the robotic instrument to which it is attached; $u_{\text {rob/hldr2 }}$ is the uncertainty in the orientation of the robotic instrument relative to the detector holder, where the detector holder is attached to the robotic instrument; $u_{\text {hldr/dctr }}$ is the uncertainty in the orientation of a reference plane of the detector holder relative to a reference plane on the metal detector; and $\sigma_{\theta}$ is the standard deviation of the angular variation of the orientation of the outer surface of the metal detector relative to the plane of the detector coil. In the LPU given in Eq. (3), it is assumed that the variation in all the parameters is normally distributed and that the measurement uncertainty contributors are derived from many independent measurements.

It is edifying to estimate the value of Eq. (3) and then compare this value to simulations that demonstrate the effect of angular deviation on exemplar detectability. To this end, we now estimate the uncertainty contributions to Eq. (3). The $\sigma_{\theta}$ describes manufacture reproducibility. For HHMDs, and for quality manufacturing (per ISO 9001), it would be reasonable to expect that $\sigma_{\theta}$ would be less than $5^{\circ}$ in each orthogonal direction of a Cartesian coordinate system. For HWMDs, by their very nature of being pliable to conform to being worn, $\sigma_{\Theta}$ may be greater than $10^{\circ}$ and variable between different users or tests. For each of the other five measurement uncertainty contributors, based on observation and measurement, it is expected they contribute $5^{\circ}$ or less each per axis. To simplify our estimate of angular uncertainty, we will consider only one coordinate axis and combine the uncertainties from the other two axes onto one axis. We will also assign an equal uncertainty for each of the contributors shown in Eq. (1) of $5^{\circ}$. More than likely, the angular uncertainty for some of these contributions will be greater. The total uncertainty, $u_{\text {oreint }}$, using this approximation is about $12^{\circ}$ for a $67 \%$ confidence interval, or about $24^{\circ}$ for a $95 \%$ confidence interval. This angular uncertainty may allow the metal detector to be mischaracterized as having greater detection capability than it actually has.

For example, consider a knife exemplar that is designated as a "small" size threat for a given orientation, namely that orientation that provides the least coupling to the magnetic field generated by the metal detector. Figure 1 shows six different orthogonal orientations that a knife exemplar can have with respect to the metal detector (shown by the loop). The arrow in Fig. 1 shows the direction of the motion of the exemplar relative to the metal detector. Each of these orthogonal orientations are arranged such that one surface of the test object is parallel to the plane of the coil. It is important to note that detecting the threat at the least coupling orientation is the basis for defining the metal detector as being able to detect the designated size threats. So, if, during testing, the orientation of the "small" size test object is off from the ideal value $\left(0^{\circ}\right)$, it will present a larger surface for coupling to the magnetic field and, consequently, provide a signal commensurate to that from a larger "medium" size test object. An alarm would then be generated by the metal detector and this alarm would be incorrectly attributed to the "small" size test object. As an example, the effect of orientation on coupling for the knife exemplar is shown in Table 1. If spherical exemplars are used, this orientation problem vanishes.

Table 1. The relative coupling of a knife exemplar for the orientations shown in Fig. 1, with orientation $\mathrm{C}$ as the reference. The values were obtained using electromagnetic simulation.

\begin{tabular}{|l|c|c|c|c|c|c|}
\hline Orientation & A & B & C & D & E & F \\
\hline Relative coupling & 0.6777 & 0.0919 & 1.000 & 0.1284 & 0.1464 & 0.0517 \\
\hline
\end{tabular}

\section{Simulation of Exemplar Coupling}

In this section, we provide a relative comparison of the coupling of the metal detector's magnetic field to current exemplars as a function of their orientation and to the proposed spherical exemplars. The coupling values are derived from electromagnetic simulation to avoid the relatively large errors that may be encountered using experimental values. The simulation describes the inductive coupling to the test object, which is the fundamental basis upon which a detection alarm is generated. There are several methods for detecting and augmenting the signals received by the detector to generate this alarm. It is not the purpose of this work to simulate or compare these proprietary methods. However, the trend of the simulations was qualitatively verified by experiments. It should also be mentioned that demagnetization will affect the 


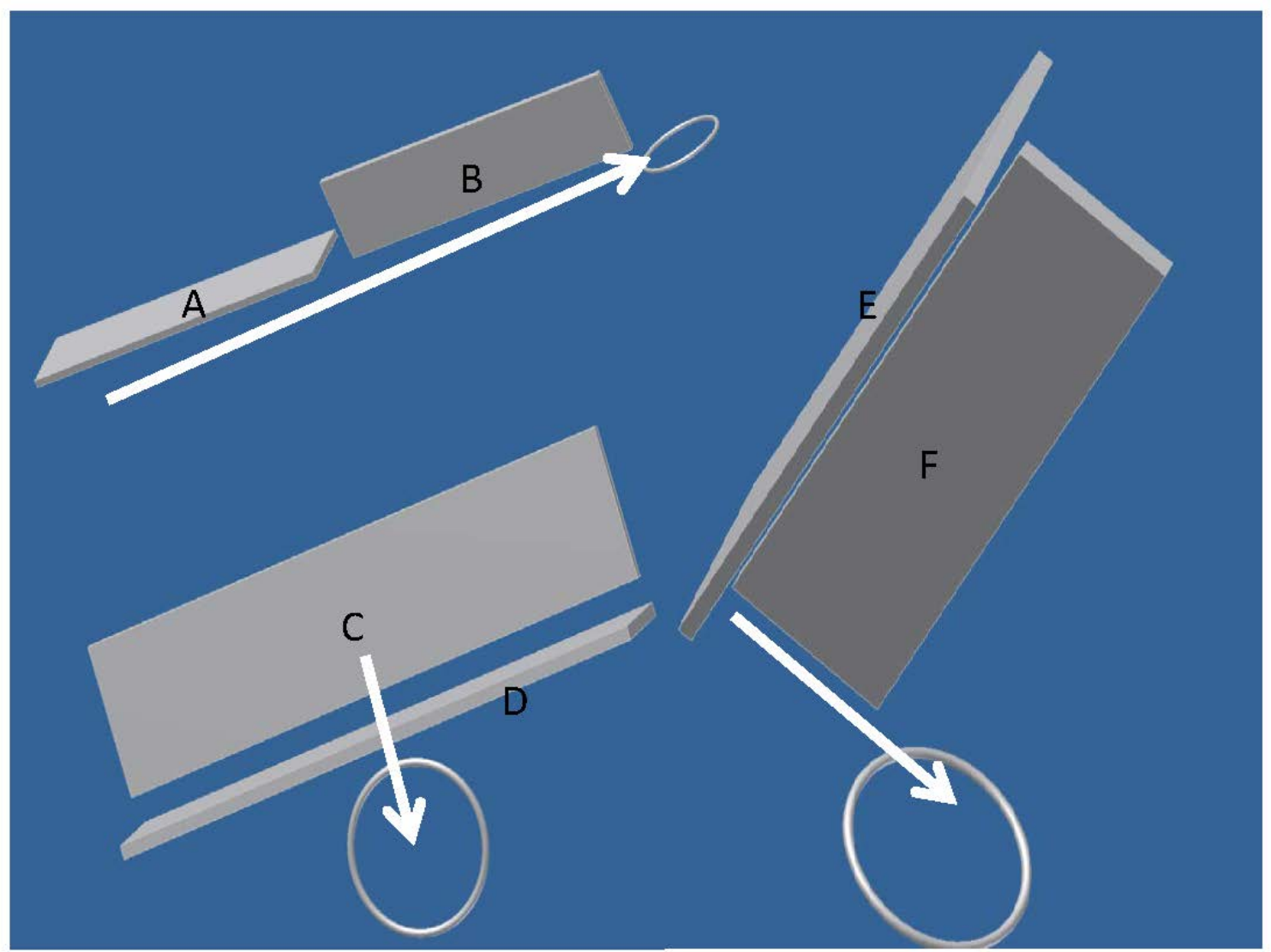

Fig. 1. The possible unique orthogonal orientations of a thin rectangular prism that can be presented to a metal detector, which is represented by a loop. The white arrows show the direction of motion of the test object relative to the loop.

detectability of test object depending on its geometry and orientation with respect to the applied magnetic field, and this has been calculated for simple geometries [3-5]. The simulation software did take into account demagnetization as shown by the simulations of a spherical test object and comparison of those simulation results to the numerical results of [4] (data not shown).

Spherical test objects are currently used by certain manufacturers to track performance of a metal detector over time and to perform a reproducible comparative evaluation of metal detector models. We propose the use of spherical test objects in a minimum performance standard because they will allow more accurate predictions of the performance of a metal detector than will actual threat objects or exemplars that attempt to resemble the threat object. This does not mean that metal detectors should not be tested against agency-specific threats. But when performing such field tests, the user of the equipment should be cautioned regarding the effect of orientational uncertainty and the potential to erroneously qualify a metal detector fit for purpose when it is not.

All simulations were performed using a commercially-available magnetic field simulator that uses the finite element method. The circuit for the simulation comprises a $50 \mathrm{kHz}$ current source and a single-loop coil, where the coil is a solid-copper wire with a $1 \mathrm{~mm}$ diameter cross section and an inside loop diameter of $20 \mathrm{~mm}$. The distance between the loop and the nearest surface of the exemplar is $1 \mathrm{~mm}$, as this is a representative operating distance for the metal detector. A change in the loop characteristics and drive signal will change the relative coupling for a given exemplar. However, for comparisons between different exemplars, a common and fixed set of loop characteristics and a fixed drive signal are necessary to observe the effect of the exemplar on coupling between it and the metal detector. Magnetic anisotropy of ferromagnetic materials was not simulated because this effect is anticipated to be very much smaller than 
the geometry effects that are currently seen with the use of simulated threat objects. The coupling between the coil and test object is measured as a change in the inductance of the coil circuit due to the presence of the test object.

The possible unique orthogonal orientations of the knife exemplar (a simple rectangular prism) that can be presented to a metal detector are shown in Fig. 1. Each of these orthogonal orientations are arranged such that one surface of the test object is parallel to the plane of the coil. The separation between the coil and the surface closest to the plane of the coil was fixed, as this represents actual use. In practical applications using a HWMD or HHMD, the most likely orientations that will be presented to the metal detector are orientations A and C (see Fig. 1). All the test objects shown in Appendix A similarly have no more than two orientations that will be presented to the HWMD or HHMD during actual use of the metal detector. The orientations that provide the least coupling for the knife exemplar are B, D, E, and F, with orientation $\mathrm{F}$ representing the least coupling condition. The relative coupling for these orientations are shown in Table 1. Figure 2 shows the relative increase of coupling as the knife exemplar is rotated from orientation $\mathrm{F}$ to orientation $\mathrm{E}$. Note that the relative coupling of orientation $\mathrm{E}$ is almost three times greater than that of orientation F.

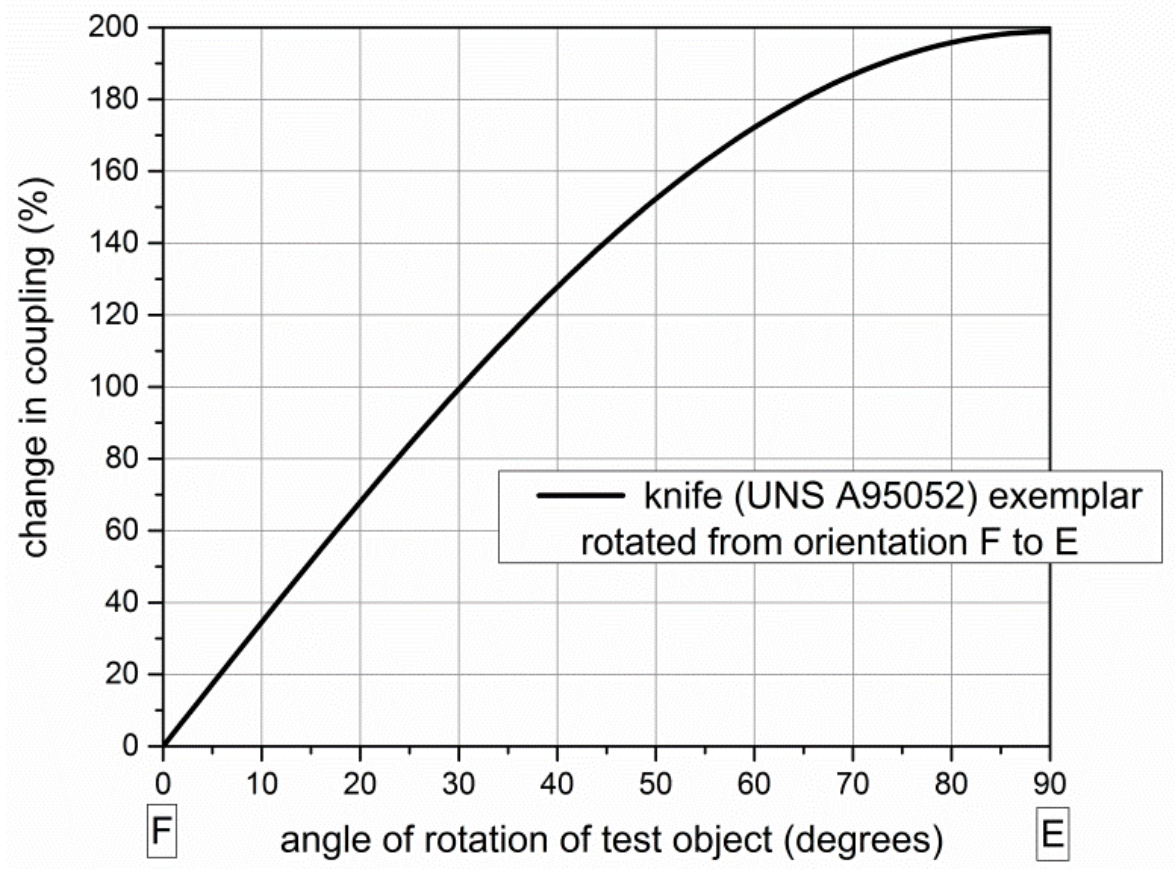

Fig. 2. Relative increase in coupling as aluminum (UNS A95052) knife exemplar is rotated from orientation F to orientation E.

Figure 3 shows the effect on the measured inductance change in the wire loop by rotating a nonferromagnetic test object from one orientation to another. For example, the knife exemplars are rotated from orientation B to orientation A (see Fig. 1). The test objects were rotated between the orientations providing maximum and minimum coupling. The presence of the nonferromagnetic test object in the coil circuit effectively adds a parallel inductance as the eddy currents induced in the test object will generate a secondary magnetic field opposing that of the source. The result is a reduction in the inductance of the loop circuit. The metals used for the different test objects are given in Appendix B. Also shown in Fig. 3 is the inductance change due to the presence of a solid aluminum (UNS A96061) sphere. Figure 4 exhibits the same information as shown in Fig. 3 but with a close up of the smaller rotation angles and sphere radii. The information displayed in Figs. 3 and 4 can be used to determine the radius of a sphere that can be used to replace the test objects shown in Appendix A. 


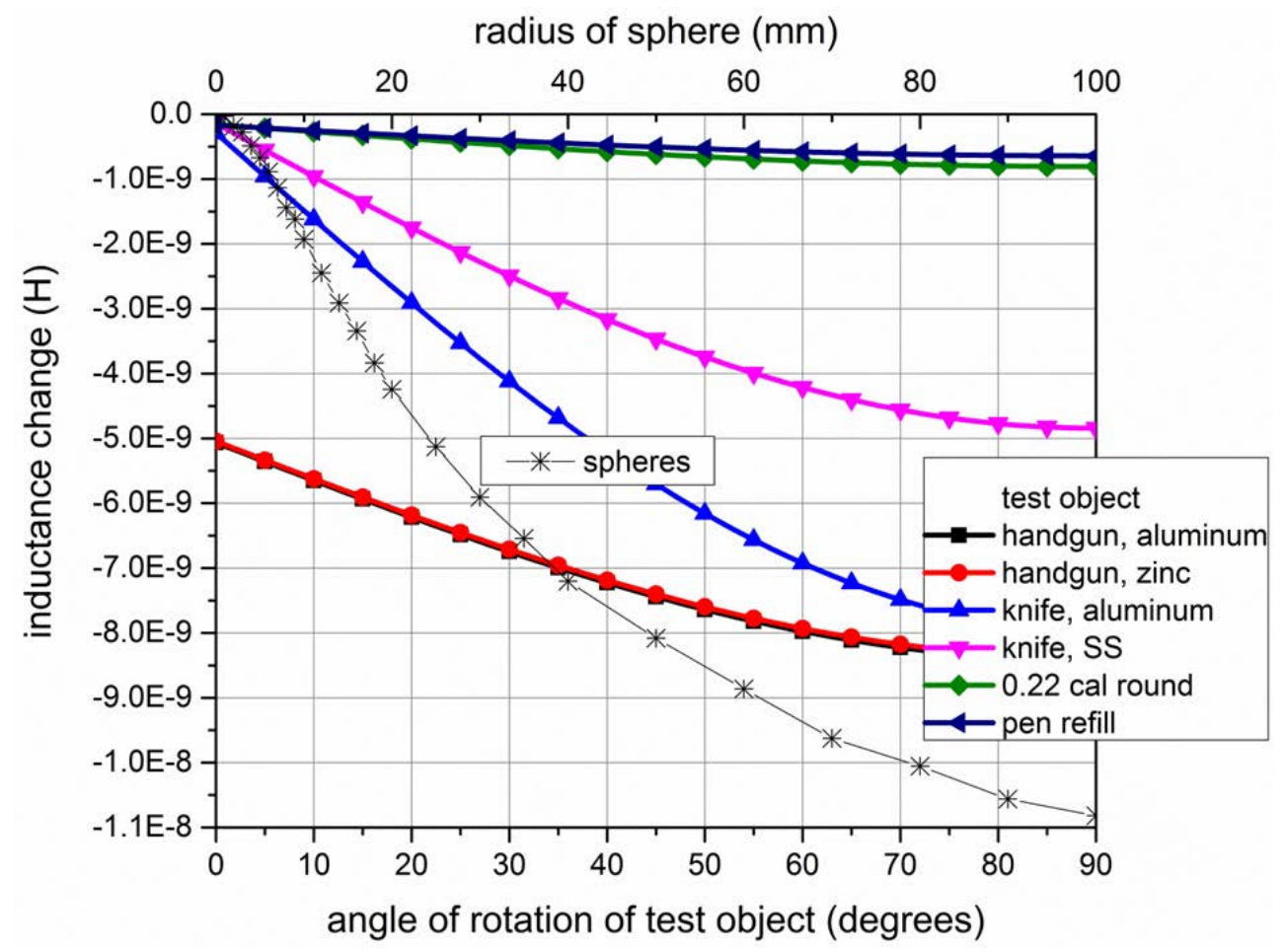

Fig. 3. Inductance change caused by rotation of nonferromagnetic test objects (lower $\mathrm{x}$ axis) and changing sphere radius (upper $\mathrm{x}$ axis). Sphere is UNS A96061.

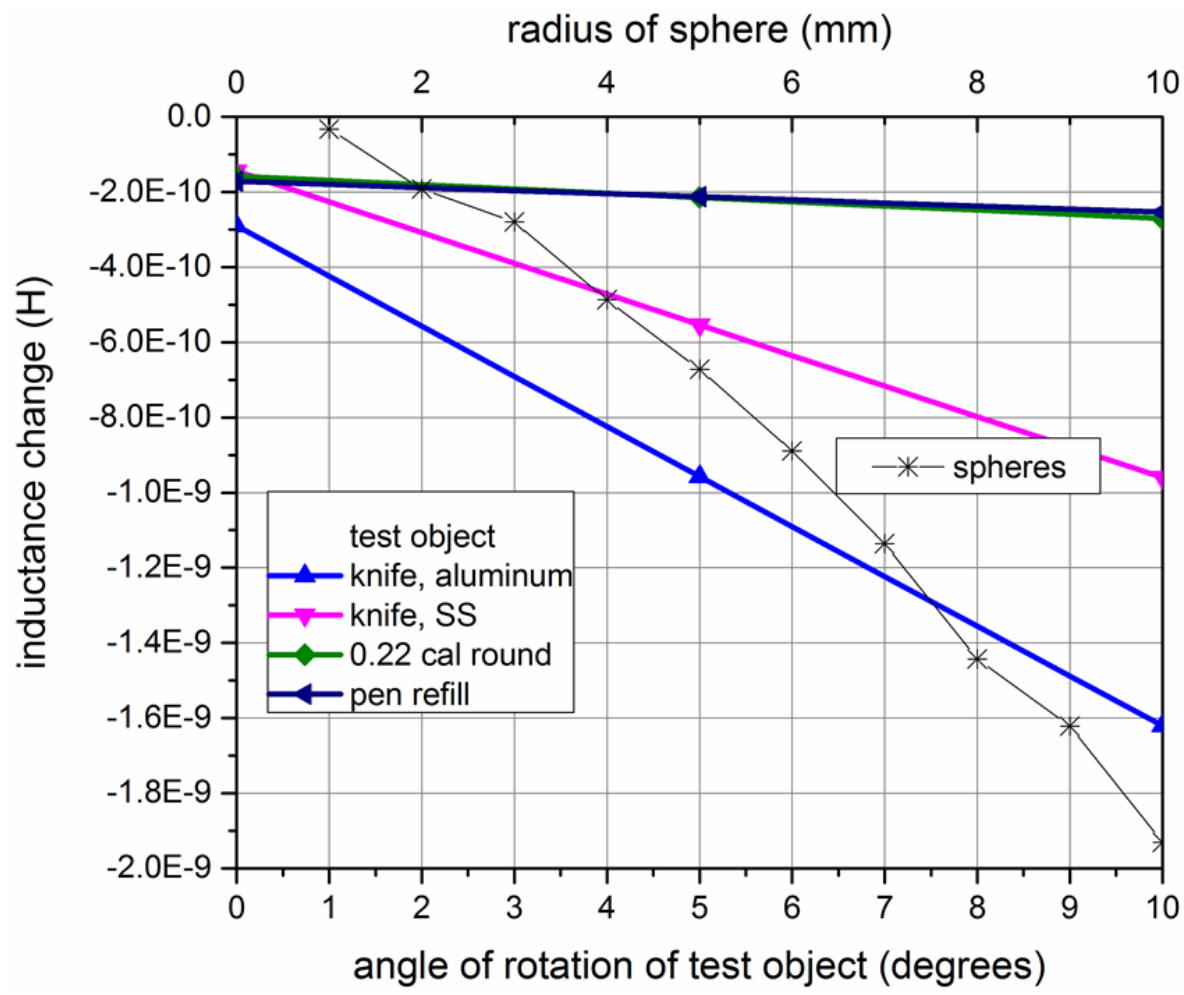

Fig. 4. Close up of Fig. 3 that shows the smaller angles of the test objects and the smaller diameter spheres. Sphere is UNS A96061. 
Figure 5 shows the effect of rotating a ferromagnetic test object from one orientation to another. The test objects were rotated between the orientations providing maximum and minimum coupling. The difference between the effect of a nonferromagnetic and a ferromagnetic test object is the phase of the induced eddy current. For the nonferromagnetic case, increasing the interaction between the test object and the magnetic field (see Figs. 3 and 4) decreases system inductance, whereas for the ferromagnetic case the opposite is true. The metals used for the different test objects are given in Appendix B. Also shown in Fig. 5 is the inductance change due to the presence of a solid steel (UNS G10180) sphere.

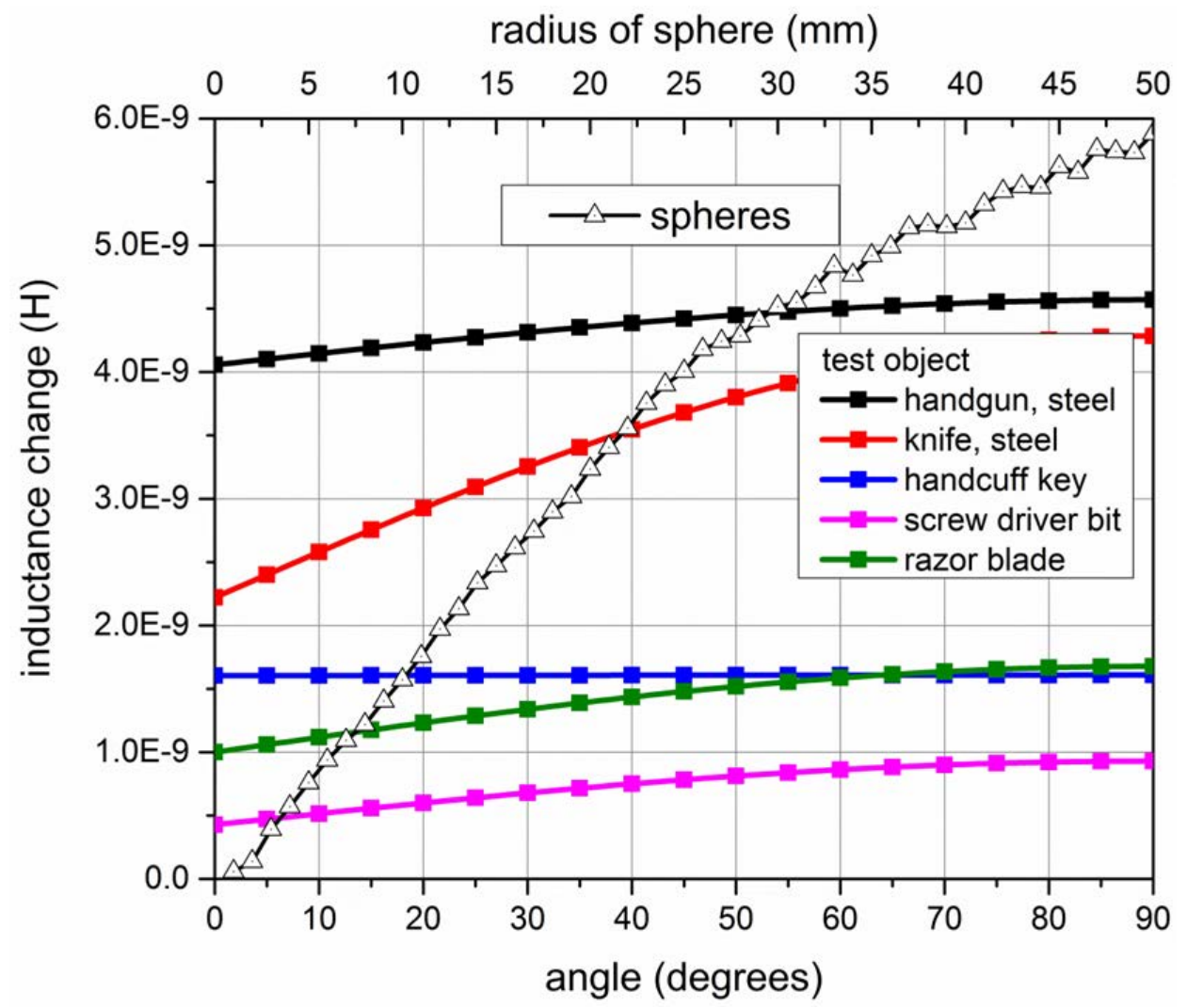

Fig. 5. Inductance change cause by rotation of ferromagnetic test objects (lower $\mathrm{x}$ axis) and changing sphere radius (upper $\mathrm{x}$ axis). Sphere is UNS G10180.

Figure 6 shows an example of the effect on inductance from variation in the steel (UNS G10180) sphere's radius, $r$, around its nominal value. In this figure, the nominal radius is $10 \mathrm{~mm}$. For this example, the percent change in inductance is about four times the change in the radius. Consequently, it is important to ensure that the tolerances placed on machining the sphere will allow the exemplar to be used to test the metal detector performance to the required accuracy.

Figure 7 shows an example of the effect on inductance from variation of the metal's electrical conductivity, $\sigma$, around its nominal value. In this figure, the nominal value of $\sigma$ is around $2.02 \times 10^{7} \mathrm{~S} / \mathrm{m}$, which is typical for aluminum. For this example, the percent change in inductance is about one-tenth of the change in $\sigma$. Consequently, it is not essential to require tolerances on electrical conductivity of less than about $10 \%$ of nominal value because smaller tolerances may make material acceptance difficult without commensurate improvement in measurement uncertainty. 


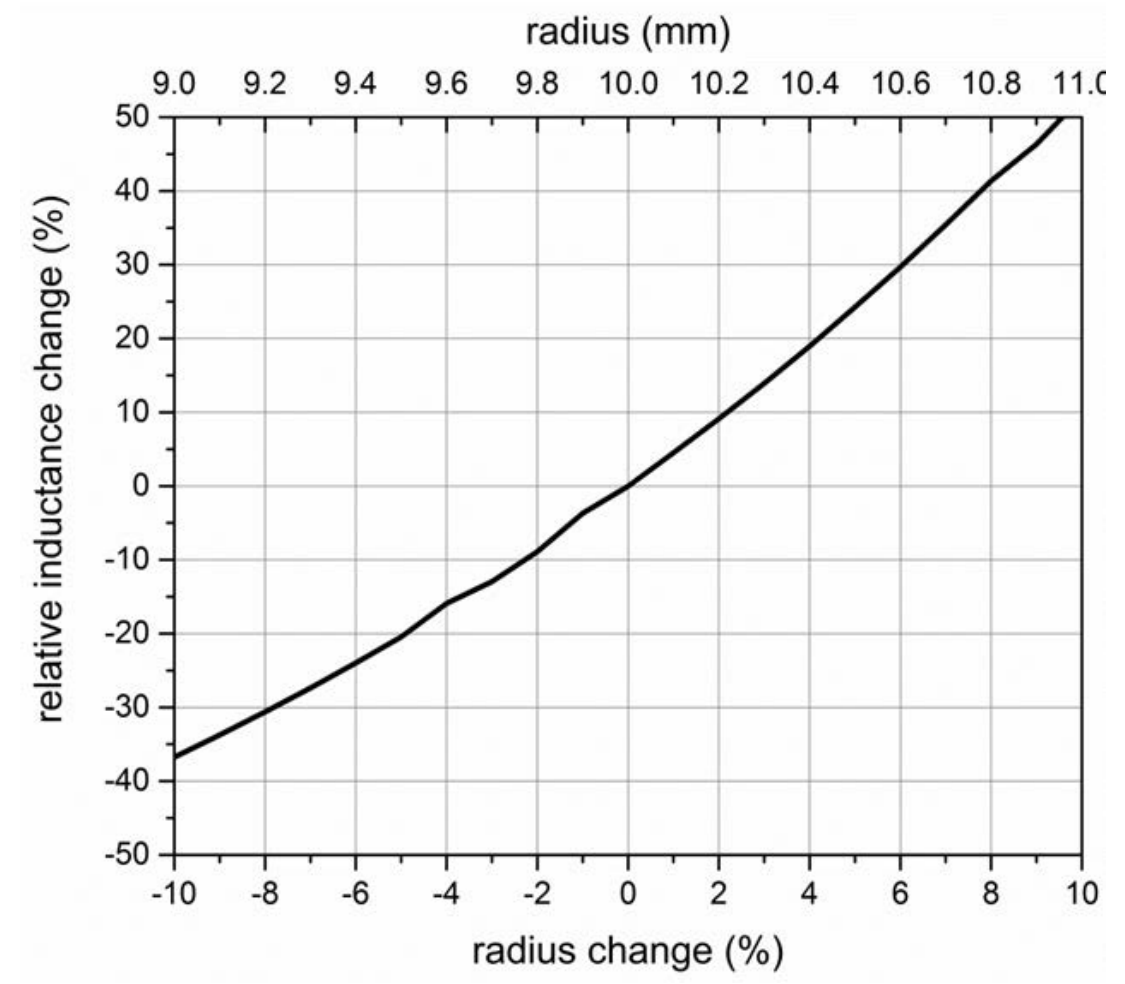

Fig. 6. A simulation showing the effect of variations of the sphere radius to its inductance.

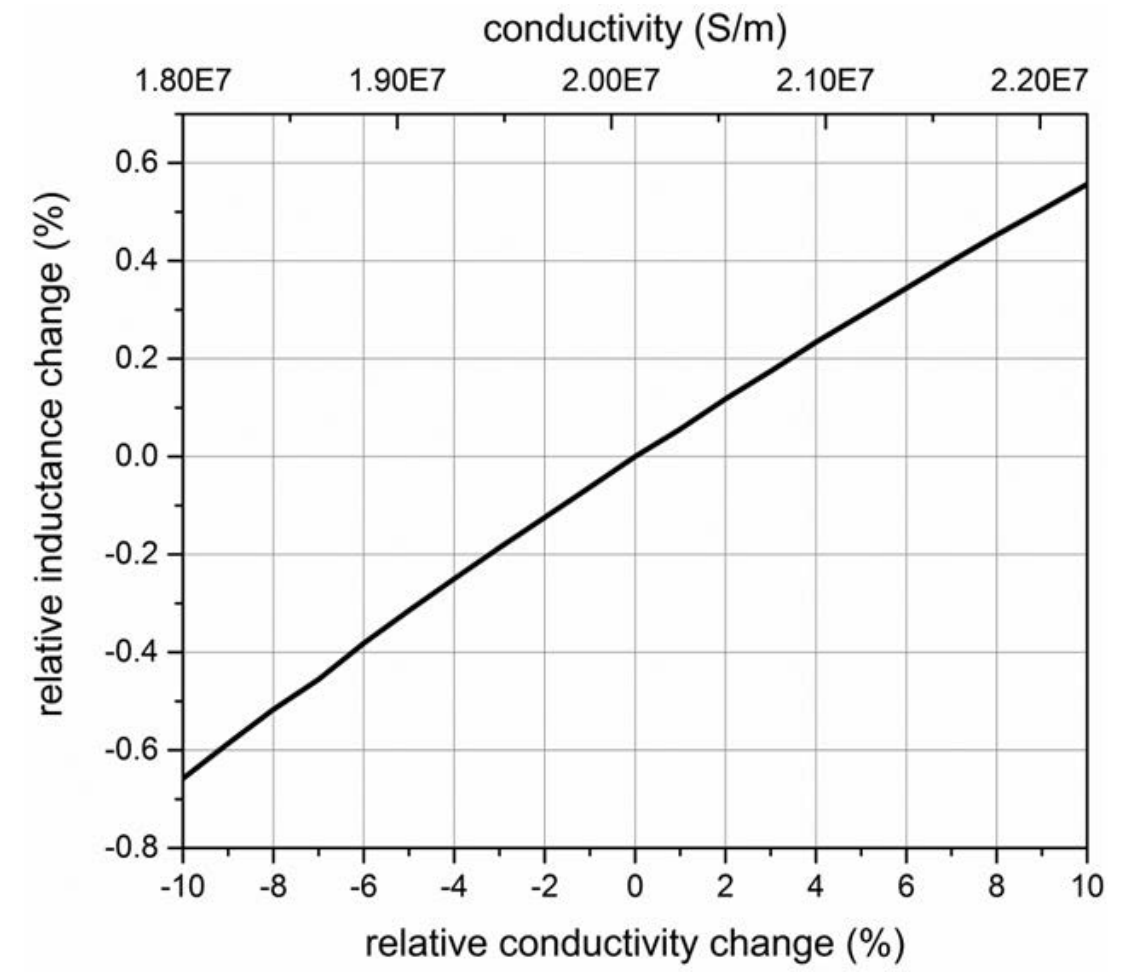

Fig. 7. A simulation showing the effect of variations on $\sigma$ to the inductance caused by the presence of the sphere. 
Figure 8 shows an example of the effect on inductance from variation of the metal's magnetic permeability, $\mu$, around its nominal value. In this figure, the nominal $\mu$ is $8 \pi \times 10^{-5} \mathrm{H} / \mathrm{m}$ (relative $\mu$ of 200), which is within the range of typical ferromagnetic steels. For this example, the percent change in inductance is equal to or less than the change in $\mu$. It is important to ensure that the tolerances placed on $\mu$ are as small as possible to ensure the exemplar can be used to test the metal detector performance to the required accuracy. However, $\mu$ may change with time depending on its exposure to temperature changes and mechanical shock. The simulations also show that, as a percentage of the value of $\mu$, variations in smaller values of $\mu$ cause larger variations in the inductance than do variations in larger values of $\mu$. Furthermore, the effect of variations in permeability on inductive coupling is less for the sphere than other test objects.

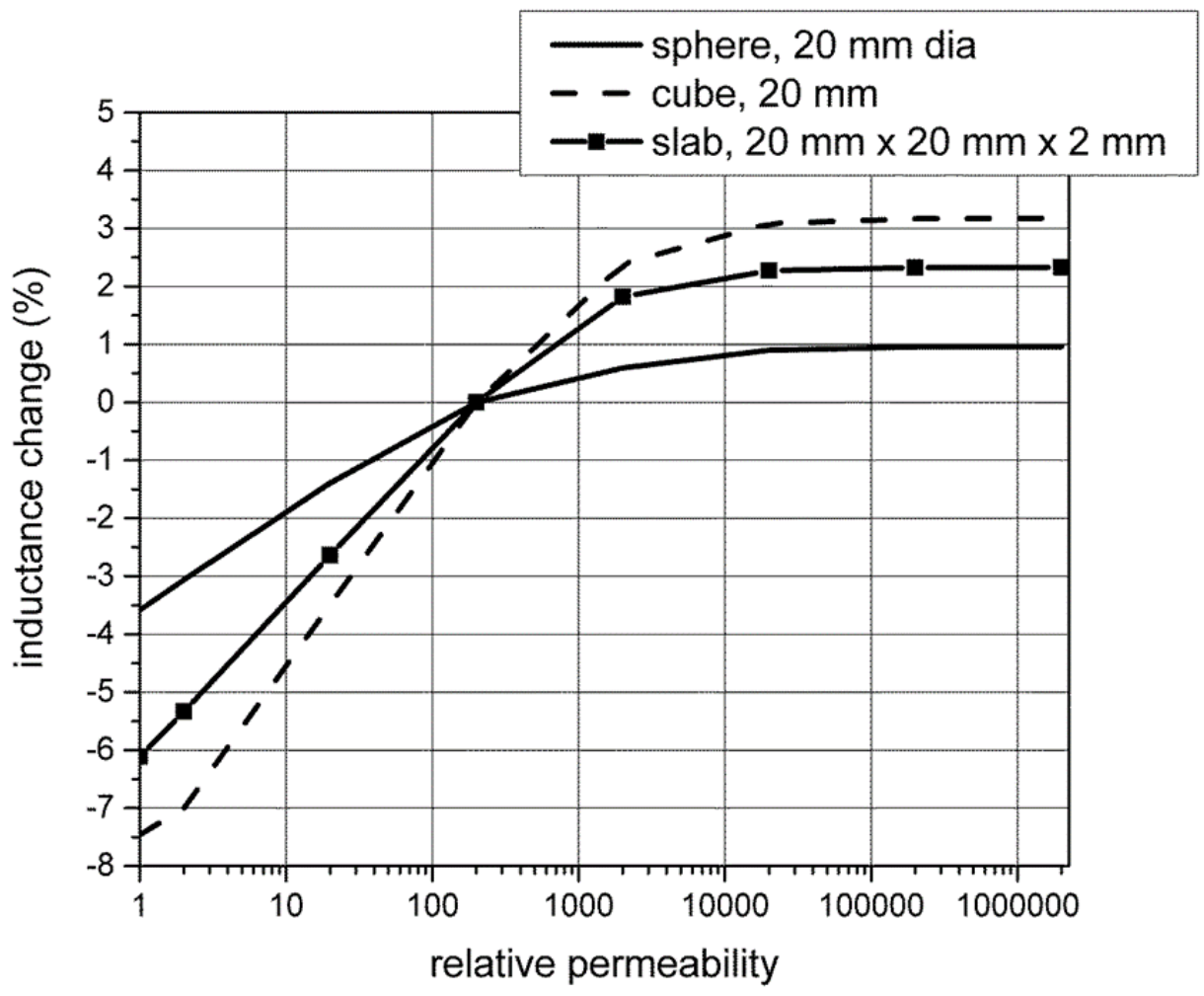

Fig. 8. A simulation showing the change in inductance caused by changes in the relative permeability, $\mu_{r}$, for different test objects. The inductance change is relative to that for $\mu_{r}=200$. The slab was oriented so that its large surface was parallel to the plane of the coil. The cube was oriented so that a surface was parallel to the plane of the coil.

\section{Summary}

The detection signature of an arbitrarily-shaped metal object from a metal detector will depend on the mass, geometry, and orientation with respect to the magnetic field, electrical conductivity, and magnetic permeability of the test object. For the nationally-standardized test objects currently used for metal detector assessment, the largest contributor to the uncertainty in detector assessment is the orientation of the test object with respect to the magnetic field generated by the metal detector. Spherical test objects do not have an orientational uncertainty contribution and, thus, will provide more reliable, reproducible, and accurate assessment of the detection performance of metal detectors than will the current test objects. Because the magnetic permeability affects the response of the metal detector, it is necessary to have spherical test objects made of both ferromagnetic and nonferromagnetic metals. Therefore, to replace the 11 current nonspherical test objects will require eight spherical test objects: two each for each object size 
classification, where one is made from a ferromagnetic metal and the other from a nonferromagnetic metal. The use of spherical test objects can result in an acceptable international test method for the minimally acceptable detection performance of hand-worn and hand-held metal detectors. However, each unique security application may require testing for the unique threats of that application.

\section{Appendix A}

Mechanical drawings of test objects in the current National Institute of Justice metal detector standards [2] and that have been proposed for an ASTM metal detector standard (Figs. A1-A7). These test objects were developed by the National Institute of Standards and Technology.

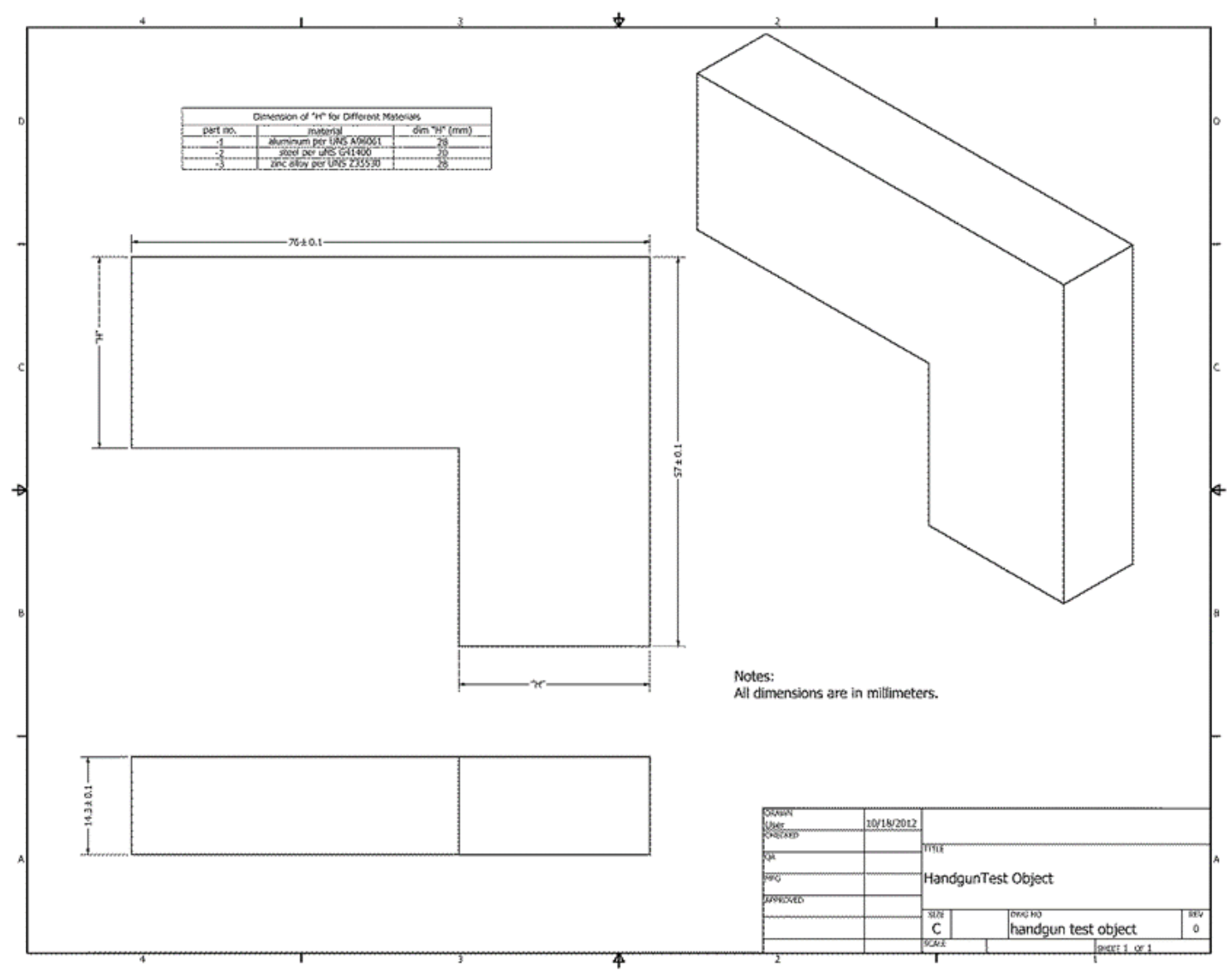

Fig. A1. Handgun exemplar currently proposed in draft ASTM standard for HHWD. This is considered a large-size test object. Different materials and sizes are used for the construction of this exemplar. 


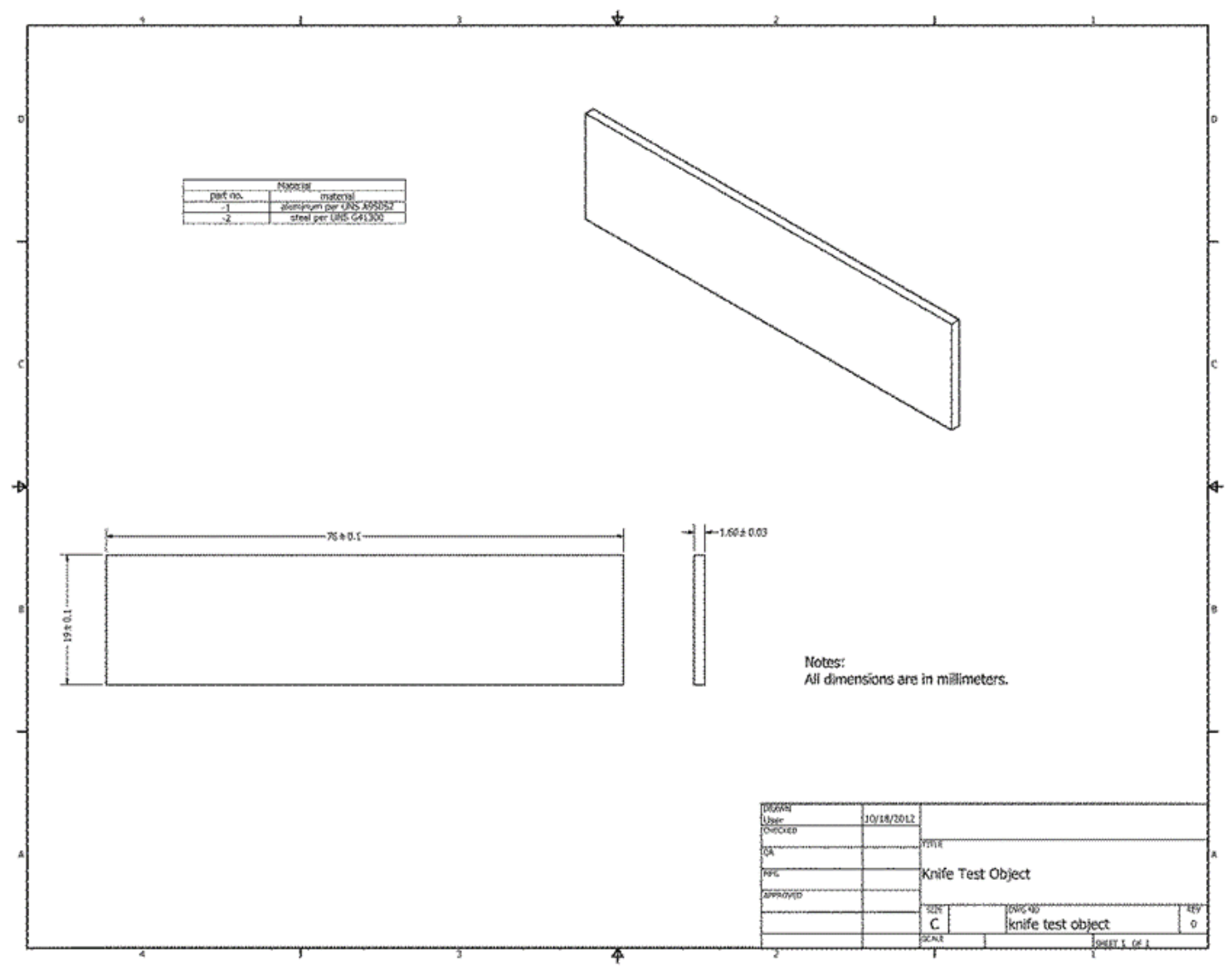

Fig. A2. The knife exemplar is a simple rectangular prism. Different materials are used to construct copies of this exemplar. 


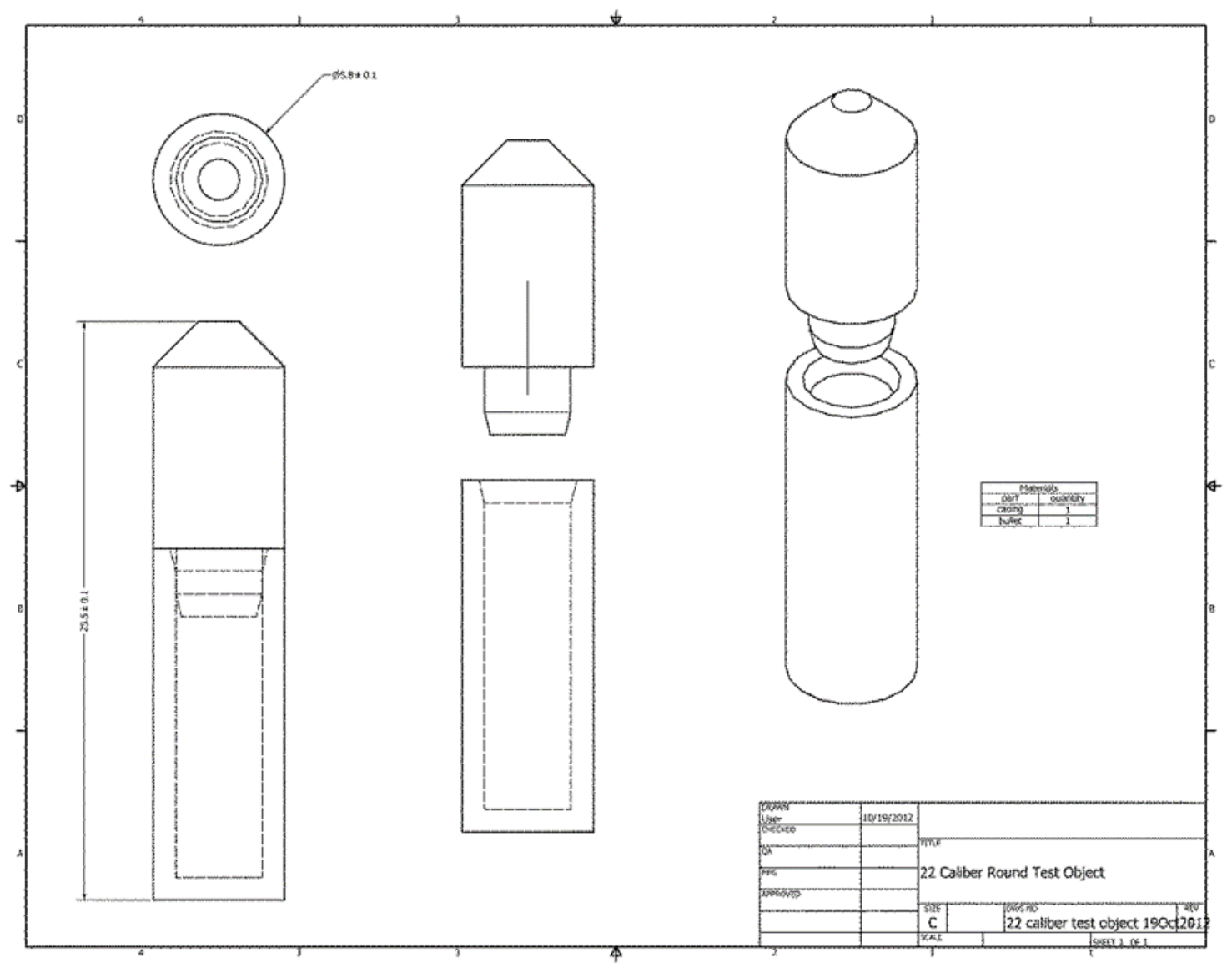

Fig. A3. The 0.22 caliber round is a small-sized test object. It comprises a lead bullet and a brass case. 


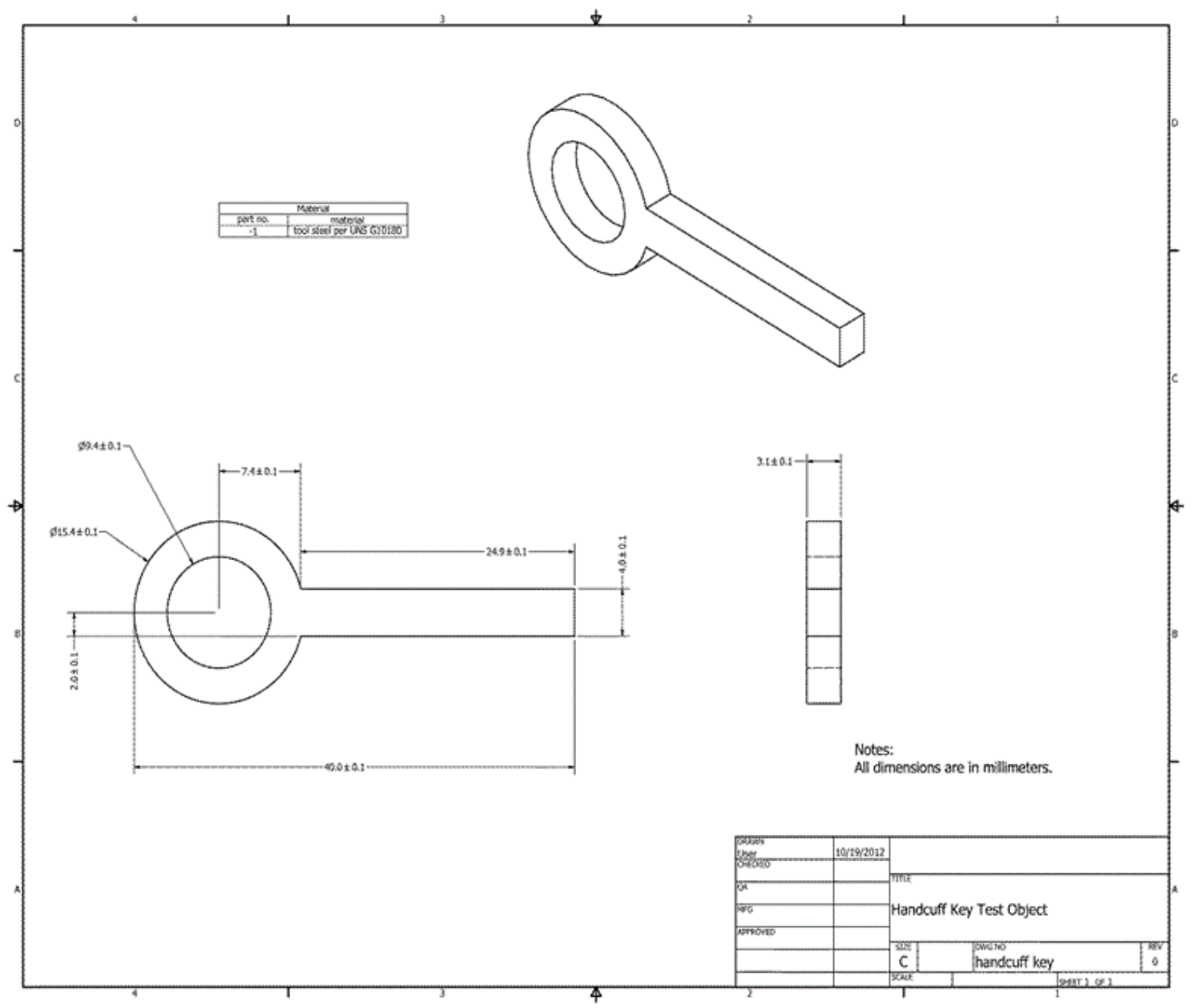

Fig. A4. A handcuff key is a small-sized test object. 


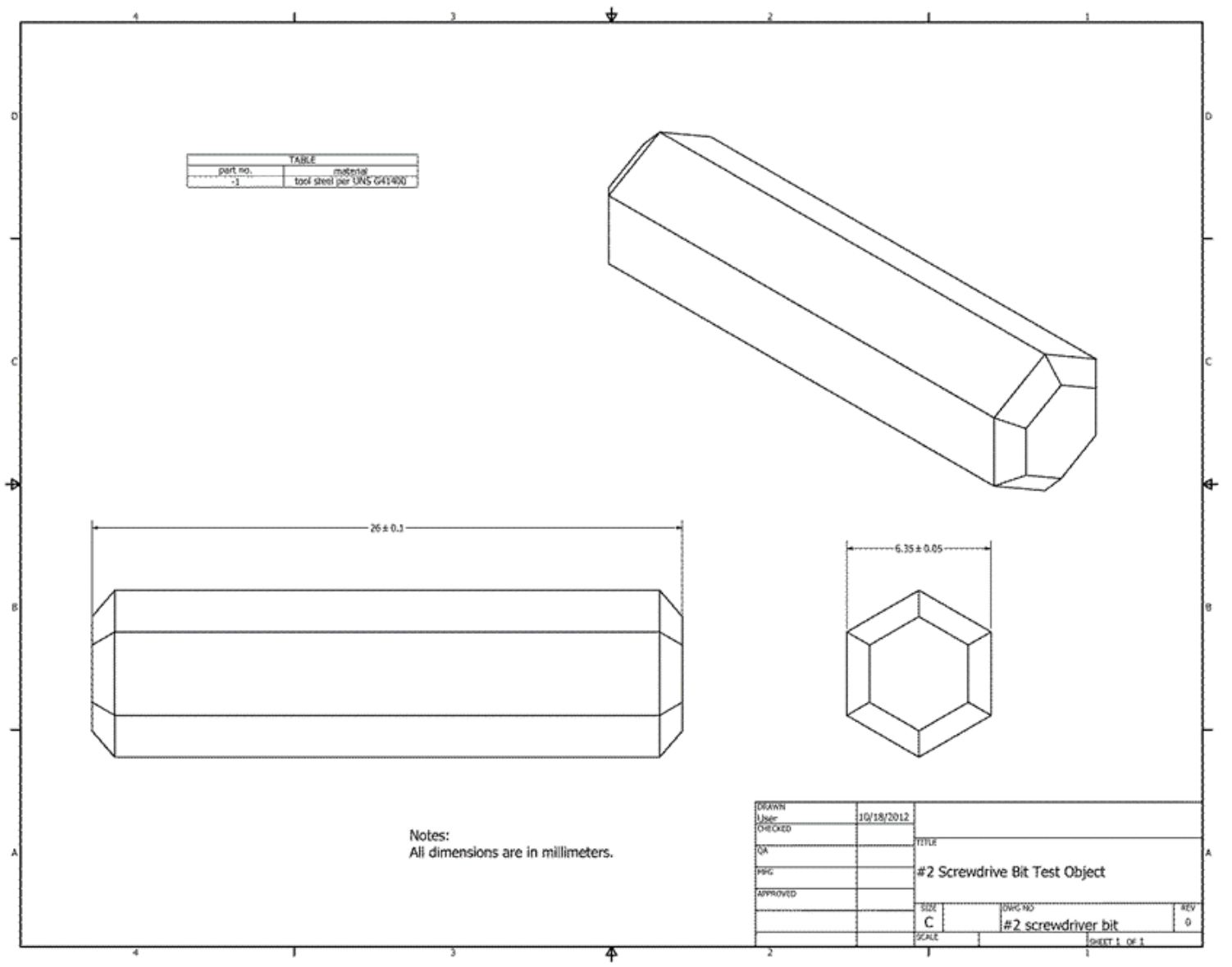

Fig. A5. The hexagonally-shaped steel piece is a small-sized test object intended to emulate \#2 Phillips screw driver bits that are often found in a prison setting as the tip of an inmate-fashioned spike or "shiv." 


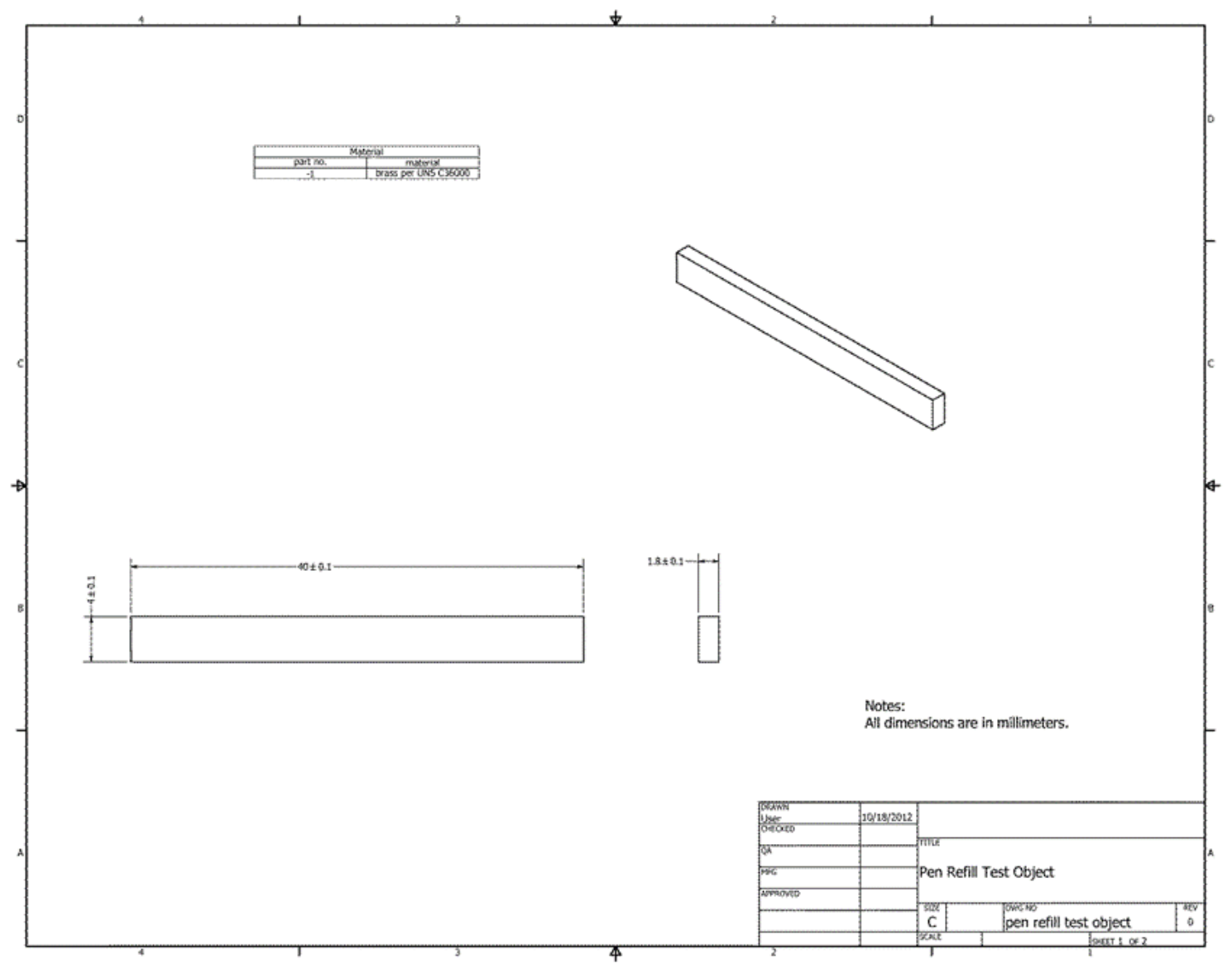

Fig. A6. This rectangular prism of brass is an exemplar intended to emulate the electromagnetic characteristics of pen refill, which are used in a prison setting to pick locks, such as those of handcuffs. This exemplar is considered a very-small-sized test object. 


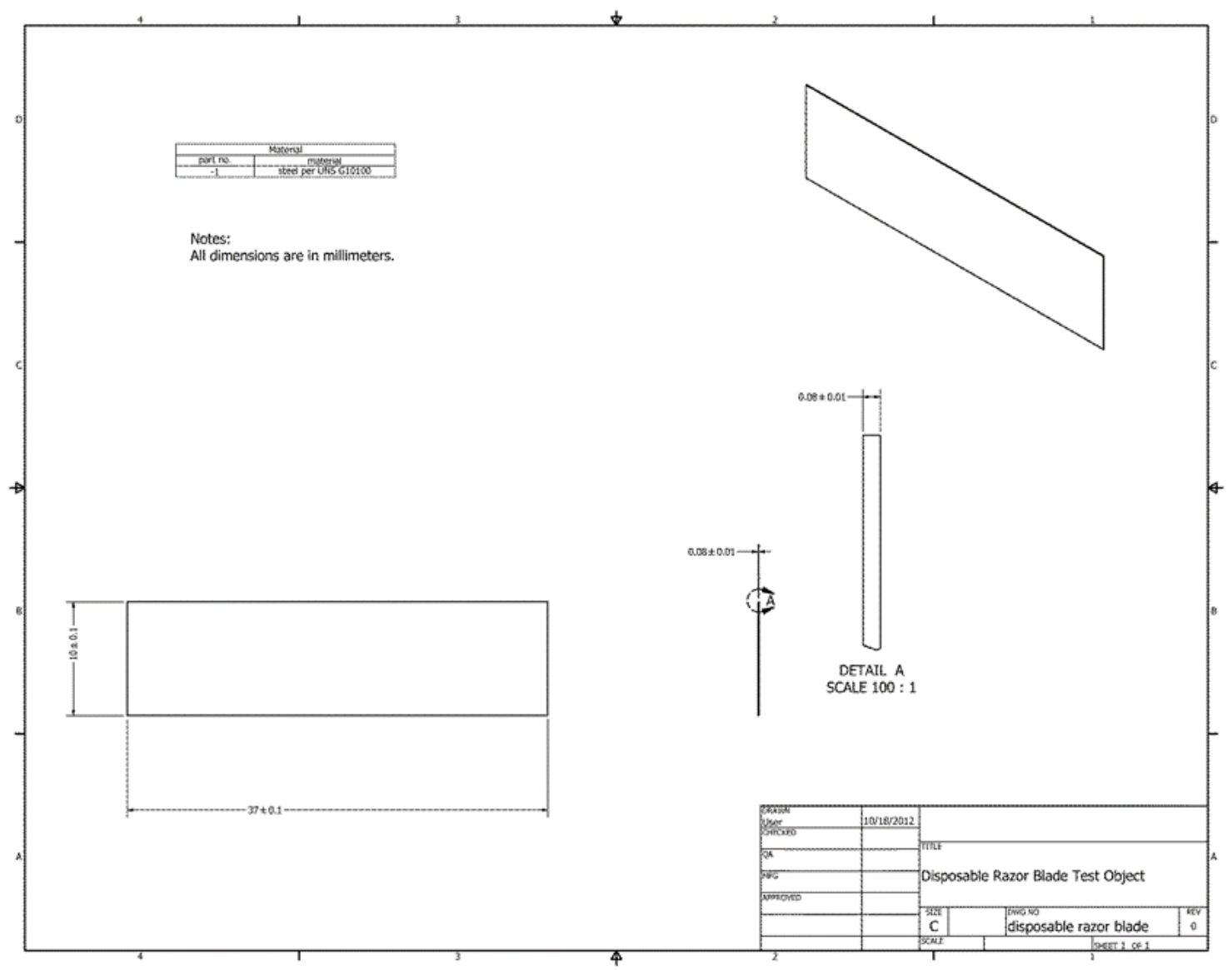

Fig. A7. This rectangular prism emulates a disposable razor blade, which in a prison setting is used as blades in inmate-fashioned knives. This is a very-small-sized test object. 


\section{Appendix B}

Materials and electromagnetic quantities for the test objects used in the current NIJ metal detector standards [2]. Electrical conductivity and magnetic permeability were measured using NIST-developed tools, as described in [6] and [7].

\begin{tabular}{|c|c|c|c|c|c|c|c|}
\hline \multirow[t]{2}{*}{ Object size } & \multirow[t]{2}{*}{ Test object } & \multirow[t]{2}{*}{ Metal } & \multirow[t]{2}{*}{$\begin{array}{c}\text { UNS } \\
\text { designation }\end{array}$} & \multicolumn{2}{|c|}{$\begin{array}{l}\text { Electrical conductivity [6] } \\
\qquad(\mathrm{S} / \mathrm{m})\end{array}$} & \multicolumn{2}{|c|}{$\begin{array}{l}\text { Relative magnetic } \\
\text { permeability [7] }\end{array}$} \\
\hline & & & & mean & tolerance & mean & tolerance \\
\hline \multirow[t]{3}{*}{ Large } & Handgun & Aluminum & A96061 & $2.78 \times 10^{7}$ & $3.2 \times 10^{6}$ & 1 & N/A \\
\hline & Handgun & Steel & G41400 & $4.49 \times 10^{6}$ & $5.9 \times 10^{5}$ & 64 & 17 \\
\hline & Handgun & Zinc & Z35530 & $2.512 \times 10^{7}$ & $1.5 \times 10^{6}$ & 1 & N/A \\
\hline \multirow[t]{2}{*}{ Medium } & Knife & Aluminum & A95052 & $2.02 \times 10^{7}$ & $2.2 \times 10^{6}$ & 1 & N/A \\
\hline & Knife & Steel & G41300 & $4.28 \times 10^{6}$ & $5.9 \times 10^{5}$ & 60 & 27 \\
\hline \multirow[t]{6}{*}{ Small } & Handcuff key & Steel & G10180 & $4.15 \times 10^{6}$ & $9.3 \times 10^{5}$ & $270[8]$ & - \\
\hline & Knife, nonmagnetic & Steel & S30400 & $1.41 \times 10^{6}$ & $4 \times 10^{4}$ & 1.05 [9] & 0.02 \\
\hline & Cartridge, 22 calibre & & & & & & \\
\hline & bullet & Lead & L50045 & $4.84 \times 10^{6}[10]$ & - & 1 & N/A \\
\hline & case & Brass & C26000 & $1.543 \times 10^{7}$ & $7.7 \times 10^{5}$ & 1 & N/A \\
\hline & Screwdriver bit & Steel & G41400 & $4.49 \times 10^{6}$ & $5.9 \times 10^{5}$ & 64 & 17 \\
\hline \multirow[t]{2}{*}{ Very small } & Pen refill & Brass & С36000 & $1.297 \times 10^{7}$ & $5.8 \times 10^{5}$ & 1 & N/A \\
\hline & Razor blade & Steel & G10100 & $8.33 \times 10^{6}[10]$ & - & 190 [10] & - \\
\hline
\end{tabular}

\section{References}

[1] Bureau International des Poids et Mesures-Joint Committee for Guides in Metrology (2008) Evaluation of measurement data Guide to the expression of uncertainty in measurement. JCGM 100:2008. Available at http://www.bipm.org/utils/common/documents/jcgm/JCGM_100_2008_E.pdf. Accessed August 31, 2016.

[2] National Institute of Justice (2003) Hand-Held Metal Detectors for Use in Concealed Weapon and Contraband Detection. NIJ Standard 0602.02. Available at https://www.ncjrs.gov/pdffiles1/nij/200330.pdf. Accessed August 31, 2016.

[3] Osborn JA (1945) Demagnetizing Factors of the General Ellipsoid. Phys Rev 67(11-1):351-357. http://dx.doi.org/10.1103/Physrev.67.351

[4] Chen DX, Brug JA, Goldfarb RB (1991) Demagnetizing Factors for Cylinders. IEEE Trans Magn 27(4):3601-3619. http://dx.doi.org/10.1109/20.102932

[5] Aharoni A (1998) Demagnetizing factors for rectangular ferromagnetic prisms. J Appl Phys 83(6):3432-3434. http://dx.doi.org/10.1063/1.367113

[6] Janezic MD, Kaiser RF, Baker-Jarvis J, and Free G (2004) DC Conductivity Measurements of Metals (U.S. Department of Commerce, Washington, D.C.), NIST Technical Note 1531. http://dx.doi.org/10.6028/NIST.TN.1531

[7] Janezic MD, Baker-Jarvis J (2004) Relative Permeability Measurements for Metal Detector Research, (U.S. Department of Commerce, Washington, D.C.), NIST Technical Note 1532. http://dx.doi.org/10.6028/NIST.TN.1532

[8] Review of Progress in Quantitative Nondestructive Evaluation, eds Thompson DO and Chimenti DE (American Institute of Physics), Vol 25.

[9] Wilson N and Bunch P (1991) Magnetic-Permeability of Stainless-Steel for Use in Accelerator Beam Transport-Systems. In Conference Record of the 1991 IEEE Particle Accelerator Conference, Vols 1-5, pp 2322-2324. http://dx.doi.org/10.1109/Pac.1991.164953

[10] Hoburg JF, Clairmont BA, Fugate DW, Lordan RJ (1997) Comparisons of measured and calculated power frequency magnetic shielding by multilayered cylinders. IEEE T Power Deliver 12(4):1704-1710. http://dx.doi.org/10.1109/61.634194 
About the authors: Nick Paulter is an electronics engineer in the Materials Measurement Science Division at NIST's Gaithersburg campus where, in addition to being a group leader, develops and advances the metrology for various electromagnetic field/wave-based technologies that are used to sense the presence of threat items. Donald Larson a retired NIST electronics engineer who pioneered high-speed electrical and optical pulse metrology. He currently is the owner of Entegra Corporation, which is a manufacturer of high-speed electronic test instrumentation and provides engineering consultation. John Ely is retired from the Federal Bureau of Prisons where he was their chief technology specialist responsible for selecting, testing, and deploying threat detection technology in US correctional institutions. He currently provides security consultation to local and state agencies and equipment manufacturers. The National Institute of Standards and Technology is an agency of the U.S. Department of Commerce. 